\title{
TRAS LAS HUELLAS DEL APRENDIZAJE COOPERATIVO EN LAS APLICACIONES MÓVILES DE IDIOMAS
}

\author{
In the quest for cooperative learning in language learning mobile applications
}

\author{
Em procura das impressões da aprendizagem cooperativa nos aplicativos \\ móveis de idiomas
}

Ana María Aguilar López

Universidad de Burgos (Espanha)

amaguilar@ubu.es

\section{Resumen}

En el presente artículo analizamos seis aplicaciones móviles de idiomas para valorar en qué medida se incluye en ellas el aprendizaje colaborativo. Para ello, detectamos las posibilidades de expresión e interacción que estas incluyen y valoramos quiénes son los sujetos de las mismas, en qué lenguas se realizan esos intercambios, cómo y con qué finalidades. Los resultados demuestran que, a pesar de que las aplicaciones puedan ser concebidas como un instrumento individual, también existe en ellas la idea de incorporar a otras personas al aprendizaje del usuario y de hacerlo con diferentes finalidades, pero mayoritariamente en la lengua vehicular y no tanto en la lengua meta.

Palabras clave: Aplicaciones móviles de idiomas. Lenguas Extranjeras - Segundas Lenguas. Aprendizaje cooperativo.

\begin{abstract}
In the following article, six language learning mobile applications (apps) are analyzed to determine to what extent collaborative learning is used. In order to do that, we detected the possibilities for expression and interaction to be found in the applications and evaluated who the people involved in those exchanges are, how they take place, in which language and for what purposes. The results proved that, in spite of the applications being conceived as a personal tool, they also feature the possibility of interacting with other people for different purposes, but mostly in the user's language and not so much in the target one ${ }^{1}$.
\end{abstract}

Key words: Language learning mobile applications. Foreign Languages - Second Languages. Cooperative learning.

\footnotetext{
${ }^{1}$ Queremos dar las gracias al traductor D. Dan Calvo Santa Olalla por la altruista traducción al inglés del resumen, del título y de las palabras clave del artículo que aquí se incluyen.
} 


\section{Resumo}

No presente artigo analisamos seis aplicativos móveis de idiomas para valorizar em que medida se inclui nelas a aprendizagem colaborativa. Para isso, detectamos as possibilidades de expressão e interacção que estas incluem e valorizamos quem são os sujeitos das mesmas, em que línguas se realizam esses intercâmbios, como e com que finalidades. Os resultados demonstram que, apesar de que os aplicativos possam ser concebidos como um instrumento individual, também existe neles a ideia de incorporar a outras pessoas à aprendizagem do utente e do fazer com diferentes finalidades, mas maioritariamente na língua vehicular e não tanto na língua meta.

Palavras-chave: Aplicativos móveis de idiomas. Línguas Estrangeiras - Segundas Línguas. Aprendizagem cooperativa.

\section{INTRODUCIÓN: MARCO TEÓRICO}

Las nuevas tecnologías se han colado en nuestra cotidianeidad llegando a formar parte inherente de ella, las posibilidades de ofrecer servicios mediante estas aumentan día a día y, como era de esperar, el aprendizaje no ha permanecido de espaldas a este hecho, antes bien, ha encontrado en ellas un potente aliado para la formación del usuario.

El aprendizaje de lenguas mediante tecnología ubicua es un campo que está en desarrollo y continua reestructuración, pero también es un campo que goza de mucho éxito entre los usuarios por cuanto permite fomentar un aprendizaje autónomo y se adapta al nivel y al tiempo del que disponen los usuarios. Este querer fomentar el aprendizaje autónomo y centrarse en el usuario de una manera tan individualizada, desprovisto del grupo clase (o no contando necesariamente con él, al menos a priori), pudiera parecer irreconciliable con el aprendizaje cooperativo, pero ¿realmente es así?

El aprendizaje cooperativo ha sido definido por Smith (1996, p. 71) como "la utilización en la enseñanza de pequeños grupos para que los alumnos trabajen juntos con el fin de maximizar el aprendizaje, tanto el propio como el de cada uno de los demás". Para Olsen y Kagan (1992) este aprendizaje se alcanza a través del intercambio de información socialmente estructurado entre los miembros del grupo; en esta situación, el estudiante no sólo es responsable de su aprendizaje, sino que también está motivado para aumentar el aprendizaje de los demás miembros del grupo.

De entre los muchos autores que han tratado de especificar las características que ha de tener un aprendizaje para que sea catalogado de "cooperativo", nos quedamos con la propuesta de Johnson, Johnson y Holubec, quienes en 1999 reconocieron las siguientes: 1) la 
existencia de una interdependencia positiva que los miembros del grupo pueden percibir de forma clara y que les hace ser conscientes de que en el aprendizaje están vinculados, de manera que todos necesitan al resto, porque en tanto en cuanto cada uno logre sus objetivos individuales, puede contribuir a los objetivos del grupo, es decir, existe una suerte de acuerdo tácito asumido por todos en el que cada miembro ha de aportar y contribuir para poderse también beneficiar de lo que otros aporten; 2) la interacción como herramienta para facilitar el aprendizaje, pues mediante la comunicación entre los miembros es posible compartir conocimientos, perfilar conceptos y profundizar en ellos, ayudar y apoyar a otros miembros del grupo no solo en la comprensión y asimilación de esos conocimientos, sino también en todo lo que rodea al propio proceso de aprendizaje; 3) la responsabilidad individual que tiene cada miembro del grupo en la consecución de los objetivos del grupo, dado que el esfuerzo individual revierte en el grupal, 4) el uso frecuente de destrezas interpersonales o habilidades sociales que permitan crear ambiente propicio para generar relaciones entre los miembros del grupo y 5) la evaluación grupal a la que se somete el grupo para que cada miembro tenga oportunidad de valorar su actuación dentro del grupo y éste sea capaz de valorar el proceso identificando posibles puntos de mejora para un futuro.

A pesar de estas características, asumimos que, como ya reconocieron Johnson, Johnson y Stanne en el año 2000, el aprendizaje cooperativo no tiene una única manera de llevarse a cabo, sino que este término da cobertura a una serie de métodos y técnicas que difieren entre sí sensiblemente y que autores como Ovejero (1990) o Slavin (1990) ya han caracterizado. Entre ellos los más citados en la bibliografía suelen ser: el grupo de investigación, el aprendizaje en equipo, el ropecabezas o puzzle, aprender juntos, equipos torneo, equipos de enseñanza individualizada asistida, etc. No todos han sido creados para trabajar las mismas materias ni se han aplicado a todas, pero su técnica sí es acomodaticia, a veces incluyendo variantes, al aprendizaje de diferentes materias. Algunos de ellos responden a un modelo cooperativo más puro y otros incluyen elementos más individualistas y competitivos, pero todos ellos contemplan, eso sí, la idea de la existencia del grupo y de la expresión, comunicación o interacción entre sus integrantes para acceder al conocimiento y afianzarlo; la idea de ayudar a otros a aprender y la evaluación del grupo, muchas veces reconocida mediante un sistema de recompensas.

Son muchos los estudios que confirman las ventajas atribuidas a la inclusión del aprendizaje cooperativo en el proceso de enseñanza aprendizaje de lenguas, pero estas ventajas se han confirmado en contextos de aprendizaje en los que el grupo clase está 
constituido, de manera que ¿qué sucede con las aplicaciones móviles?, ¿consideran estas la posibilidad de formar grupos o son un material didáctico al servicio del aprendizaje en soledad?, en caso de que sí puedan constituirse grupos, ¿es posible establecer equivalencias entre grupos de aprendizaje cooperativo dentro del aula física y los grupos virtuales que no están dotados de elementos tan asociados al aprendizaje como la figura de un profesor? Para dar respuesta a estos interrogantes, en el presente artículo nos proponemos 1) examinar si existe en estas aplicaciones contacto e interacción con otras personas y, en caso de que sea así, tratar de caracterizarlos, es decir, 2) valorar en qué medida se incluyen estos contactos y comunicaciones, 3) identificar quiénes son los interlocutores y qué funciones desempeñan, 4) con qué finalidades y en qué lenguas se desarrollan estos intercambios y todo ello para, en última instancia, relacionarlo con posibles huellas del aprendizaje cooperativo.

\section{DESARROLLO}

Hemos estructurado este punto en tres epígrafes, a saber: 1) Metodología: apartado en el que especificamos y justificamos los criterios de selección de las aplicaciones que van a formar el corpus de estudio de este trabajo; 2) Recogida de datos, tras descargar todas las aplicaciones, vamos registrando la información que podemos observar en cada una de ellas que se relaciona o podría relacionarse con el aprendizaje cooperativo; y 3) Análisis de los datos: donde tratamos de interpretar los datos recogidos en el punto anterior.

\subsection{Metodología}

Al seleccionar las aplicaciones que van a convertirse en el centro de nuestro estudio, tomamos como referencia una serie de criterios, a saber: 1) han de ser aplicaciones a través de las cuales se puedan trabajar varios idiomas, es decir, que no se presenten como aplicaciones que trabajan una sola lengua, 2) no se han de anunciar como aplicaciones concebidas para trabajar en exclusividad algún aspecto concreto de la lengua o actividad de lengua (comprensión, expresión, interacción o mediación orales y escritas), sino que, al menos a priori, parezca que permiten abordar la lengua meta de manera global, y 3) deben ser aplicaciones gratuitas, si no en su totalidad, parcialmente, pues nuestra investigación se va a centrar en los contenidos gratuitos que estas ofrecen.

Hemos seleccionado estos tres criterios porque nos permiten acotar el objeto de estudio de manera clara y establecer clasificaciones de aplicaciones; así, con relación al 
primero, hemos de especificar que si tomamos como referencia el objeto de estudio, nos damos cuenta de que existen diferentes tipos de aplicaciones para aprender idiomas, unas permiten al usuario trabajar varias lenguas de manera simultánea mediante la instalación de una sola aplicación, mientras que hay aplicaciones que ayudan al usuario a trabajar una sola lengua meta, de modo que si el usuario quiere ejercitarse en tres lenguas se ha de descargar tres aplicaciones. Por otro lado, existe la posibilidad de que algunas herramientas ofrezcan ambas posibilidades, esto sucede, por ejemplo, con Babbel, Bosuu o Mondly. En nuestro análisis, incluimos aplicaciones que trabajen varias lenguas porque vivimos en una sociedad globalizada en la que cada vez es más habitual encontrar comunidades multilingües y personas plurilingües, de manera que no parece descabellado pensar que mucha gente quiera practicar más de una lengua.

Otro criterio diferenciador se relaciona con los contenidos o destrezas que nos permiten trabajar las aplicaciones de idiomas, a este respecto, cabe señalar que hay aplicaciones que se conciben para que el usuario practique la lengua de un modo general $\mathrm{u}$ holístico y otras que se centran en un aspecto de la lengua, como por ejemplo el vocabulario, la conjugación verbal, etc.; o en una actividad de la lengua específica como pueda ser la mediación escrita o la comprensión.

Si atendemos a un criterio económico, las aplicaciones destinadas a la enseñanza de idiomas, al igual que las de otros campos, pueden ser gratuitas o no, aunque, por lo general, las que se anuncian como "gratuitas" ofrecen la posibilidad de contratar ciertos productos por un coste adicional o de poder acceder a más pruebas incluso a certificados 2 si hay una suscripción, cuyo importe está en función de la duración que dicha suscripción tenga.

Para localizar las aplicaciones de nuestro estudio, escribimos en Play Store de Google Play la ecuación: "aprender idiomas gratis"3 de entre los resultados que arroja la búsqueda, seleccionamos los seis primeros que cumplen con los anteriores requisitos, estos resultados se corresponden con las aplicaciones de Duolingo, Babbel, Memrise, 50 Languages, Busuu y Mondly.

Dado que, como ya hemos especificado, entre los objetivos de esta investigación están identificar, examinar y caracterizar posibles intercambios comunicativos que se incluyen en estas aplicaciones, para, en última instancia, valorar si es posible hablar de la presencia de aprendizaje cooperativo; tras la selección de las aplicaciones, buscamos y extraemos de Play

\footnotetext{
${ }^{2}$ Este es el caso por ejemplo de Busuu, que ofrece a los suscriptores Premiun la opción de conseguir un certificado oficial de nivel avalado por McGraw Hill.

${ }^{3}$ Los resultados corresponden a la búsqueda realizada el 1 de febrero de 2017.
} 
Store dos tipos de datos: por un lado aquellos que puedan servirnos de presentación para cada aplicación, en concreto nos fijamos en la última actualización que han tenido, su origen y el número de veces que han sido descargadas; y por otro lado datos que nos inciten a pensar en la posibilidad de comunicación o interacción, en este sentido nos fijamos en las valoraciones que hacen los usuarios de cada aplicación, si se reconoce en la información adicional de Play Store la presencia de elementos interactivos dentro de la aplicación y la mención explícita a redes sociales asociadas a cada una.

Una vez que hemos registrado estos datos que se ofrecen al usuario desde Play Store, procedemos a descargarnos cada una de estas aplicaciones, para comprobar in situ, mediante la manipulación de la aplicación, incluida la realización de ejercicios, qué posibilidades de expresión e interacción hay y quiénes son los interlocutores.

\subsection{Recogida de datos}

Con la intención de tener una información general de las aplicaciones, registramos los datos, ya especificados en la metodología, que aparecen disponibles en Play Store para cada una de ellas, con esos datos confeccionamos la Tabla 1 
PROGRAMA DE PÓS-GRADUAÇÃO EM COMUNICAÇÃO DA UNIVERSIDADE FEDERAL DE SANTA MARIA

Tabla 1 - Parte de la información disponible en Play Store de Google Play a 1 de febrero de 2017.

\begin{tabular}{|c|c|c|c|c|c|c|c|}
\hline $\begin{array}{c}\text { NOMBRE } \\
\text { APP. }\end{array}$ & $\begin{array}{c}\text { ÚLTIMA } \\
\text { ACTUALIZACIÓN }\end{array}$ & ORIGEN & $\begin{array}{l}\text { DESCAR- } \\
\text { GAS }\end{array}$ & $\begin{array}{l}\text { VALORA- } \\
\text { CIÓN }\end{array}$ & $\begin{array}{c}\mathrm{N}^{\circ} \mathrm{DE} \\
\text { OPINIONES }\end{array}$ & $\begin{array}{c}\text { ELEMENTOS } \\
\text { INTERACTIVOS }\end{array}$ & $\begin{array}{l}\text { SITIO WEB } \\
\text { ASOCIADO }\end{array}$ \\
\hline Duolingo & 27 de enero de 2017 & $\begin{array}{l}\text { Pittsburgh } \\
\text { EE.UU. }\end{array}$ & $\begin{array}{c}\text { Más de } 50 \\
\text { millones }\end{array}$ & 4,7 & 3.963 .013 & $\begin{array}{l}\text { Los usuarios } \\
\text { interactúan }\end{array}$ & $\begin{array}{c}\text { https://es.duolingo.com } \\
\text { Facebook } \\
\text { Twitter } \\
\text { Google }+\end{array}$ \\
\hline Babbel & $\begin{array}{l}22 \text { de diciembre de } \\
2016\end{array}$ & $\begin{array}{c}\text { Berlín } \\
\text { Alemania }\end{array}$ & $\begin{array}{c}\text { Más de } 10 \\
\text { millones }\end{array}$ & 4.1 & 146,213 & Compras digitales & $\begin{array}{c}\text { www.babbel.com } \\
\text { vídeos en youtube } \\
\text { Facebook } \\
\text { Twitter } \\
\text { Instagram }\end{array}$ \\
\hline Memrise & 1 de febrero de 2017 & $\begin{array}{l}\text { Londres } \\
\text { Inglaterra }\end{array}$ & $\begin{array}{l}\text { Más de } 5 \\
\text { millones }\end{array}$ & 4,7 & 543.741 & $\begin{array}{c}\text { Los usuarios } \\
\text { interactúan Compras } \\
\text { digitales }\end{array}$ & $\begin{array}{r}\begin{array}{r}\text { Enlace a visitar su sitio } \\
\text { web }\end{array} \\
\frac{\text { https://www.memrise.c }}{\text { om/ }}\end{array}$ \\
\hline $\begin{array}{l}50 \\
\text { Langua- } \\
\text { ges }\end{array}$ & $\begin{array}{c}9 \text { de diciembre de } \\
2016\end{array}$ & $\begin{array}{l}\text { Florida } \\
\text { EE.UU. }\end{array}$ & $\begin{array}{l}\text { Más de } 5 \\
\text { millones }\end{array}$ & 4,3 & 48.765 & $\begin{array}{l}\text { No figura este } \\
\text { apartado en la } \\
\text { información } \\
\text { adicional }\end{array}$ & $\begin{array}{l}\text { Enlace a visitar su sitio } \\
\text { web } \\
\frac{\text { https://www.50languag }}{\text { es.com/ }}\end{array}$ \\
\hline Busuu & 31 de enero de 2017 & $\begin{array}{l}\text { Londres } \\
\text { Inglaterra }\end{array}$ & $\begin{array}{c}\text { Más de } 10 \\
\text { millones }\end{array}$ & 4,3 & 176.871 & $\begin{array}{l}\text { Los usuarios } \\
\text { interactúan }\end{array}$ & $\begin{array}{c}\text { https://busuu.com } \\
\text { Facebook } \\
\text { YouTube } \\
\text { Twitter } \\
\text { Blog }\end{array}$ \\
\hline Mondly & 12 de enero de 2017 & $\begin{array}{c}\text { Brasov } \\
\text { Rumanía }\end{array}$ & $\begin{array}{l}\text { Más de } \\
100.000\end{array}$ & 4,7 & 18.907 & Compras digitales & $\begin{array}{c}\begin{array}{c}\text { Enlace a visitar su sitio } \\
\text { web }\end{array} \\
\frac{\text { https://www.mondlylan }}{\text { guages.com/ }}\end{array}$ \\
\hline
\end{tabular}

Fuente: Elaboración propia. 
Dado que la información que aparece en Play Store incluye también la opinión de los usuarios, por entender que dicha opinión es una manera de expresión que los gestores o encargados de la propia aplicación pueden considerar, decidimos registrar también los datos que se relacionan con ellas y que atienden a un sistema de estrellas en el que 5 es la máxima puntuación y 1 estrella la mínima; fruto de estos datos es la Tabla 2.

Tabla 2 - Valoraciones de los usuarios según la información disponible a 1 de febrero de 2017 en Play Store de Google Play.

\begin{tabular}{lcccccc}
\hline & DUOLINGO & BABBEL & MEMRISE & $\begin{array}{c}\text { 50 LAN- } \\
\text { GUAGES }\end{array}$ & BUSUU & MONDLY \\
\hline 5 estrellas & 3.091 .908 & 87,344 & 437.472 & 33.035 & 109.636 & 15.306 \\
\hline estrellas & 663.808 & 26,429 & 84.916 & 7.050 & 35.388 & 2.379 \\
\hline estrellas & 112.693 & 10,532 & 11.510 & 3.619 & 14.371 & 555 \\
\hline estrellas & 36.030 & 6,003 & 3.546 & 1.716 & 5.848 & 211 \\
\hline estrella & 58.574 & 15,905 & 6.297 & 3.345 & 11.628 & 456 \\
\hline
\end{tabular}

Fuente: Elaboración propia.

Pasamos a continuación a registrar la información que hemos recogido de cada aplicación una vez descargadas e instaladas.

\subsubsection{Duolingo}

Tras registrarnos, desde Duolingo tenemos la posibilidad de hacer una prueba de clasificación o de nivel, lo cual puede entenderse como una manera de ajustarse a las necesidades del usuario. Al terminar la prueba, se le comunica al aprendiz el nivel que ha alcanzado de entre los contemplados por Duolingo ${ }^{4}$ y se le da el siguiente mensaje: “¡Fantástico! ¡Has subido de nivel! ¡Ahora puedes participar en las discusiones! Dirígete al foro para interactuar con otros estudiantes".

Si abrimos el "Foro", vemos que otros aprendices de la misma lengua que hayamos seleccionado nosotros $\mathrm{u}$ otra han abierto diferentes líneas de discusión que pueden relacionarse con cualquier tema de aprendizaje de la lengua: desde gramática o léxico, a

\footnotetext{
4 No hemos encontrado en Duolingo referencias explícitas a niveles que puedan ser reconocidos como "oficiales" en Estados Unidos, lugar de creación de la aplicación, o Europa (MCERL), más bien, parece que son niveles que la propia aplicación ha creado para un uso interno.
} 
fonética, cine o enlaces para acceder a conversaciones entre nativos de la lengua meta o solicitud de usuarios para conversar en la lengua meta, pasando por el propio uso de la aplicación y ofertas que ésta puede hacer de sus productos de manera puntual.

En estos foros, son los usuarios los que muchas veces actúan como verdaderos docentes, pues no sólo responden con explicaciones a las dudas de sus compañeros o profundizan sobre temas gramaticales, léxicos, etc. que consideran importantes aunque otros no hayan preguntado, sino que también dan consejos sobre el proceso de aprendizaje, refuerzan la motivación de los compañeros, aportan información adicional de recursos, de la cultura meta, de la aplicación y su manejo, etc. Es posible seguir estos foros porque, cuando hemos de elegir un idioma, la aplicación tiene en cuenta la lengua materna ${ }^{5}$ del usuario y así si por ejemplo decimos que hablamos español y queremos aprender francés, la aplicación nos remite a francés para hablantes de español, de este modo, es previsible que las dudas que surjan en los foros tengan en cuenta la idiosincrasia de la lengua materna de los usuarios, al más puro estilo del análisis contrastivo.

En cualquier momento podemos añadir nuevos idiomas de aprendizaje y podemos consultar la información que Duolingo nos facilita, vg.: la cantidad de hablantes nativos que tiene esa lengua o la variedad que vamos a trabajar; al hilo de esta información se especifica quiénes son los "Colaboradores del curso", es decir, aquellos usuarios, nativos o aprendices, de ese mismo nivel u otro, que han contribuido, motu proprio, a los foros dando explicaciones sobre temas relacionados con la lengua meta sin necesidad de que hayan sido preguntados. Así mismo, si las explicaciones que estos colaboradores ofrecen nos resultan interesantes, atractivas, claras, etc., es posible seguirlos, como también se puede seguir a otros usuarios.

En la barra de herramientas que aparece en la parte inferior de la pantalla, hay un icono formado por dos personas y la etiqueta "Amigos", a través de esta sección, podemos agregar a nuestros amigos y, según nos dice la aplicación, competir con ellos, aunque nos surge la pregunta de si es "contra" más que "con", pues se habla de competir y no de colaborar. Al lado de este icono, aparece otro que incluye la etiqueta "Clubs", se refiere a clubes que podemos crear o a los que podemos unirnos. Para crearlos, es suficiente con buscar un nombre, seleccionar una de las insignias disponibles y clicar sobre "Crear", seguidamente Duolingo nos facilita un código que podemos compartir con nuestros amigos para que se unan a ese club. Si lo que queremos es ingresar en alguno ya existente, hemos de introducir un

\footnotetext{
${ }^{5}$ En realidad la aplicación tampoco la llama lengua materna y podríamos introducir cualquier idioma en el que nos sintamos competentes, se trata en última instancia de encontrar una lengua vehicular o puente que sirva para la transmisión de nuevas explicaciones y contenidos de la lengua meta.
} 
código que alguien, un amigo, previamente nos haya facilitado. La propia aplicación inserta información en estos clubes sobre los progresos que van alcanzando los miembros que los conforman.

Sin necesidad de estar incluidos en un club, Duolingo puede enviarnos notificaciones que sirvan para avisarnos de que tenemos que practicar, para informarnos de si tenemos un nuevo seguidor o si alguien nos supera en la cantidad de puntos que hayamos acumulado, aunque en última instancia es elección del usuario activar o no la recepción de estas notificaciones.

Si buscamos entre los ejemplos que componen los ejercicios, encontramos que cuando completamos uno y comprobamos si es correcta o no la respuesta que damos, aparece un pequeño icono con una bandera ondeante sobre el que si clicamos, surge la posibilidad de reportar un problema, es decir, de especificar si es que hay algún error en el ejercicio, en la traducción, etc., además de la bandera, aparece en casi todos los ejemplos un bocadillo de cómic que indica que ese ejemplo concreto tiene un foro de discusión. Una vez más, los usuarios exponen aquí dudas y conocimientos más o menos exactos sobre cada ejemplo.

Según la web https://www.duolingo.com/help, Duolingo incorpora un sistema de puntos que los usuarios van obteniendo cada vez que, por ejemplo, traducen oraciones o acaban una lección, pero también cuando califican traducciones de otros usuarios. Aunque nosotros no hemos encontrado esta opción de calificar traducciones de otros usuarios durante el uso que hemos hecho de la aplicación.

\subsubsection{Babbel}

Tras registrarnos, el sistema nos envía un email a la cuenta de correo que hayamos facilitado; al abrirlo el usuario se encuentra con la bienvenida a Babbel y la petición de que confirme el registro clicando sobre un espacio creado a tal efecto. Cuando lo confirmamos, se abre el enlace https://www.home.babbel.com/dashboard, en el rincón derecho superior de la pantalla que surge, podemos leer el reclamo de "Aprende en grupo". Al presionar sobre la opción que ahí se da de "Invita a tus amigos", accedemos a un mensaje en el que se nos facilita un enlace personal y en el que se nos informa de que si recomendamos Babbel a nuestros amigos y ellos crean una cuenta a través de ese enlace, nuestros amigos recibirán una semana de acceso gratuito a los contenidos que no lo son y nosotros recibiremos una semana de eso mismo por cada amigo que haya creado una cuenta a través del enlace que hemos mandado. Esto puede ser realmente un buen aliciente, pues Babbel organiza todos sus 
contenidos en cursos que incluyen un número variado de lecciones, pero de todas ellas sólo la primera de cada curso es de libre acceso.

En la aplicación de Babbel encontramos un apartado similar al de la web, pero en este caso se denomina "Invitar a amigos" y se accede a él si clicamos sobre el tercer símbolo que aparece en la barra de herramientas de la parte superior de la pantalla, es entonces cuando se despliegan varias opciones entre las que figura "Invitar a amigos", si entramos ahí, la aplicación también da al usuario la posibilidad de que nuestros amigos se unan al mundo Babbel a través de Google +, sms o vía email.

Otro punto que aparece en este despegable es el de "Ayuda", al clicar sobre él, el usuario se encuentra con la posibilidad de comunicarse con quienes están al cargo de la aplicación mediante dos preguntas: “¿Cómo te sientes?” y “¿Qué tipo de pregunta tienes?”, para que el usuario conteste a la primera de ellas, la aplicación le brinda la opción de elegir uno entre tres iconos que se corresponden con una cara de gesto algo preocupado, una cara con gesto neutro y una cara sonriente. Del mismo modo, para que el usuario dé respuesta a la segunda cuestión, ha de elegir, antes de especificar su duda, entre tres posibles iconos, uno. El primero de ellos se asocia a una duda de tipo técnico, el segundo a una consulta relacionada con el pago y el tercero a consultas de otra índole. Tras clasificar nuestra duda, podemos escribir en qué consiste y detallar lo que consideremos oportuno. En los tres casos, el procedimiento es el mismo, pinchamos sobre el icono que corresponda de nuestro estado de ánimo y del tipo de consulta que queramos hacer, (técnica, de pago u otra índole), seguidamente surge una pantalla que indica: "Escribe aquí tu mensaje" y después hay que clicar sobre "Enviar".

Con relación a los ejercicios de la aplicación, hemos de decir que se incorporan algunos diálogos que sirven para entrenar al usuario en la interacción, exponerlo a muestras de lengua y que vea el léxico trabajado en contexto.

\subsubsection{Memrise}

La aplicación se plantea a modo de misión en la que el usuario desempeña el papel de agente que ha de entrenarse con la ayuda del Control de Misiones para desarrollar sus habilidades lingüísticas que le permitan completar una serie de misiones, todo ello al objeto de recopilar información con la que llegar al dispositivo Mariposa que ha sido robado por el antagonista de esta historia, el enemigo Frente Uniformista, y evitar de este modo que el Multiverso desaparezca. Tomando como referencia este marco, la interfaz de la aplicación 
adquiere la apariencia del mando del control de una nave espacial desde el que el usuario ha de elegir el o los idiomas que quiere trabajar. El recorrido que nuestra nave ha de hacer hasta llegar al objetivo se presenta de manera esquematizada con una línea vertical por la que ascendemos de planeta en planeta o, lo que es lo mismo, de lección en lección.

Al comienzo de cada lección, se presentan los términos que se van a trabajar, para ello se suele abordar cada palabra o unidad semántica por separado, la manera de hacerlo es mostrando su forma escrita (grafía) y fonética, es decir, cómo suena en boca de un nativo, y añadiendo una traducción literal del término a la lengua vehicular que el aprendiz haya escogido. Además de esto, cada unidad semántica va asociada a un sistema de varios "mem" que la aplicación identifica con fertilizantes que provocan que los recuerdos del aprendiz crezcan fuertes rápidamente. Los "mem" son propuestas a modo de wikis que voluntariamente hacen los usuarios de la aplicación para sí mismos y para compartir con otros aprendices con la intención de ayudar a memorizar la forma, el significado o la fonética de cada término al hilo del cual el "mem" ha sido creado6.

Si vamos realizando los ejercicios de cada lección de manera correcta, la aplicación entiende que hacemos progresos, lo que trata de fomentar mediante un sistema de recompensas basado en la acumulación de puntos, medallas y rangos. Cada medalla se asocia a un rango de una escala jerárquica válida en el mundo Memrise. Cuando completamos un nivel, podemos ver en la sección de medallas cuántas personas más lo han alcanzado en esa semana y comparar los puntos que hemos acumulado con los obtenidos por otros usuarios.

Cada rango que logremos se puede compartir con quienes se desee mediante Whatsapp, Gmail, Bluetooth, Google+, Facebook, Hangouts, etc., es decir, mediante servicios que previamente tengamos instalados en el dispositivo en el que tengamos la aplicación. Además Memrise, al igual que otras aplicaciones como Duolingo, importa de las redes sociales la figura del "seguidor" al permitir que el usuario pueda seguir a varios amigos y ver sus progresos del mismo modo que también nuestro perfil puede tener varios seguidores.

Si revisamos los ejercicios propuestos en cada unidad, vemos que algunos se agrupan en el bloque denominado "Aprende con nativos", pero dicha opción no nos sirve para comunicar con ellos, pues ésta consiste en breves grabaciones audiovisuales de nativos que pronuncian las unidades semánticas que se estén trabajando en cada lección, una vez

\footnotetext{
${ }^{6}$ Por ejemplo, para el caso de "Die Wurst" ("la salchicha" en alemán) algunos usuarios se fijan en la fonética y escriben: "Di bgrost", "Dii vurst, la W suena como una V inglesa", otro se refiere a la forma escrita diciendo "Divorcio- Salchicha" y otros incluyen imágenes.
} 
activadas, simplemente hemos de identificar de entre una serie de términos escritos, el pronunciado por el nativo.

\subsubsection{Languages}

Tras descargarnos esta aplicación y revisarla, no hemos encontrado ningún apartado en el que exista la posibilidad de comunicación ni con gestores de la aplicación ni con otros usuarios de la misma. En este sentido, lo único que hemos visto es la posibilidad de compartir vía correo electrónico, Facebook, Twitter o cualquier otra aplicación que tengamos instalada en el dispositivo, la cantidad de puntos que hemos conseguido a medida que vamos dando respuestas correctas a los ejercicios propuestos por la aplicación.

\subsubsection{Busuu}

Una vez que nos registramos en Busuu, podemos acceder a diferentes niveles, desde A1- a B2, de los estipulados por el Marco Común Europeo de Referencia para las Lenguas (MCERL) (2002), cada uno de ellos se trabaja a través de diferente cantidad de lecciones. Las lecciones se presentan con contenidos y ejercicios léxicos y semánticos, gramaticales y de escritura. Es precisamente en esos ejercicios de escritura en los que encontramos una posibilidad de interacción con otros usuarios. A través de ellos se pide que se escriban oraciones breves, aunque, si el usuario se excede en el límite propuesto por la aplicación, puede seguir escribiendo.

Al mandar el ejercicio, aparece una pantalla en la que se dice: "Ejercicio enviado para que lo corrija la comunidad, ¡contribuye corrigiendo uno tú mismo!" A continuación se presenta la elección entre "Ayuda a alguien" y "No, gracias". Cuando clicamos en el primero, se nos muestra a través de cuatro pantallas un mensaje que dice: "Numerosos usuarios de todo el mundo están aprendiendo un idioma que hablas. Ayúdales corrigiendo sus ejercicios. A cambio consigue correcciones de hablantes nativos. Agrégalos como amigos para mantener el contacto e intercambiar vuestros ejercicios". En la parte inferior de la última pantalla del mensaje, el aprendiz, una vez informado, puede clicar sobre el botón: "Interactúa con otros usuarios”. Al hacerlo, surge una nueva pantalla en la que se nos pregunta: “¿Con qué idiomas te gustaría ayudar a otros usuarios? Selecciona los idiomas que hables”. A continuación, aparece una lista de idiomas, una vez que los seleccionemos, podemos clicar en la parte superior sobre la palabra: "Hecho" y entonces surgen los textos (oraciones) de otros usuarios que están aprendiendo esas lenguas en las que nosotros nos declaramos competentes y que pueden o no, ser competentes en la lengua que nosotros estemos practicando; en última instancia, nosotros decidimos los textos de quién queremos corregir, de manera que, si nos 
interesa establecer una relación más estrecha con hablantes competentes de, pongamos por caso, alemán, podemos corregir solo textos de usuarios que se reconozcan como competentes en alemán con la esperanza de poder nutrirnos más y mejor de los conocimientos del otro, quid pro quo. También es posible solicitar a algún usuario que nos corrija nuestros textos.

Para ver cómo funciona este sistema, elegimos al azar un ejemplo escrito por una persona que se declara competente en alemán que aprende español, el ejercicio de escritura de esta persona consistía en escribir una oración que describiera lo que aparece en una fotografía, en concreto la consigna que se le da para su ejercicio de escritura es: "Escribe una oración que describa cómo se siente el hombre", la fotografía muestra a un hombre con unos cascos durmiendo sobre el teclado de un ordenador portátil detrás del cual hay otro ordenador fijo. Elegimos al azar el texto de un usuario que aprende español y se declara competente en la lengua alemana, que dice: “*Él hombre está un poco cansado" y ha obtenido una respuesta de otro usuario de Venezuela que lo evalúa como perfecto dándole el máximo de 5 estrellas y escribiendo el mensaje: “¡Muy bien!”.

Si nosotros queremos apuntar algo, tenemos la opción, bien de valorar lo que ha escrito el usuario de Venezuela incluso comentar lo que ha dicho, bien de escribir un nuevo comentario que podrá ser a su vez valorado por otros correctores, bien de ambas cosas. Por ejemplo, en nuestro caso escribimos un comentario que dice: "No es "él” es "el". "ÉL" es pronombre personal masculino de tercera persona del singular (er), "EL" es artículo masculino singular (der)".

Así mismo, existe la posibilidad de que añadamos como contactos tanto a otros correctores, como a quienes acabamos de evaluar.

Busuu además incluye ejercicios con diálogos que sirven de muestra a los usuarios de interacción, aunque el aprendiz no trabaja con ellos la interacción de manera directa.

\subsubsection{Mondly}

Nada más descargarnos la aplicación, ésta nos reconoce como hablantes nativos de español y se nos pide que elijamos la lengua que queremos aprender. En esa primera pantalla ya aparece la opción de un tutorial que nos informa sobre el funcionamiento de la aplicación, al abrirlo, encontramos, entre otra información, una pantalla en la que se nos especifica: "Competición con amigos" acompañada del siguiente texto: "Evite errores para conseguir la máxima puntuación de la ronda y ser el primero entre sus competidores”. 
Si nos creamos una cuenta con Mondly, podemos hacer un perfil y conectar con nuevos amigos a través de Facebook o correo electrónico. También podemos ver una tabla de líderes de España o mundial, en la que figuran aquellos usuarios con más puntos acumulados en su aprendizaje, ya que, esta aplicación, al igual que otras, también incorpora un sistema de puntos que se obtienen a medida que se va trabajando y haciendo ejercicios correctamente.

Podemos enviar notificaciones a los gestores de la aplicación a través del icono de una bandera ondeante que aparece en el rincón superior derecho. Al clicar sobre él, podemos elegir entre dos tipos de comentarios: "He encontrado un problema" y "Necesito ayuda" en ambos casos podemos escribir un mensaje especificando el contenido con detalle.

Así mismo, Mondly incluye una serie de categorías entre las que está "Chat bot", en ella nos aparece un emoticono sonriente que nos saluda con un "Hola" y debajo de él el siguiente mensaje: "Mi nombre es Mondly, tu nuevo amigo para conversar. Juntos practicaremos conversaciones de manera divertida y relajada. Intenta contestar mis preguntas siguiendo las pistas en la pantalla". Al presionar sobre el botón de "Continuar" para acceder a este contenido, nos aparece el icono de una persona que se dirige a nosotros a través de un diálogo oral, aunque también podemos leer lo que dice; cuando nos toca intervenir, la aplicación nos da unas opciones de respuesta que se consideran válidas en esa situación comunicativa y nosotros podemos elegir la que queramos, la leemos en voz alta, (en algunos casos la aplicación nos permite que variemos ligeramente las respuestas que propone), y la grabamos para que nuestra intervención se vaya registrando de manera escrita a medida que el diálogo transcurre.

Además si accedemos a los bloques de lecciones, mediante el botón que aparece en la parte inferior con la etiqueta "Lecciones", vemos que en ellos se incluye una cantidad de lecciones, después una conversación, que va creándose con la interacción entre la aplicación y el usuario a través de grabaciones y audios y un apartado de vocabulario.

\subsection{Análisis de los datos}

Con base en la información disponible en Play Store (Tabla 1), podemos decir que una gran cantidad de personas recurren a este tipo de aplicaciones para trabajar idiomas, pues las descargas de estas aplicaciones oscilan entre los más de 50 millones de Duolingo y las 100000 de Mondly. Estas seis aplicaciones del estudio son aplicaciones que se mantienen actualizadas y que proceden tanto de Estados Unidos como de países europeos, de ahí que a 
veces se hagan eco, incluso en la información que aparece en Play Store, de los niveles prefijados por el MCERL, reconocidos oficialmente en el territorio europeo.

A juzgar por la información disponible en este espacio, de las aplicaciones analizadas, solo tres, Duolingo, Memrise y Busuu, reconocen de manera explícita que incluyen elementos interactivos para los usuarios que no se suscriben. También son tres, Duolingo, Babbel y Busuu, las aplicaciones que en Play Store remiten a redes sociales que, al menos a priori, podrían utilizarse para una telecolaboración entre los usuarios.

Teniendo en cuenta las valoraciones hechas por los usuarios, estas aplicaciones suelen dejarles bastante satisfechos, pues la puntuación menor que obtienen es la de Babbel con un 4,1 sobre 5 y la superior, con un 4,7 sobre 5, es compartida por Duolingo, Memrise y Mondly.

Un análisis más exhaustivo de la cantidad de opiniones negativas y positivas que cada una de las aplicaciones ha cosechado (Tabla 2) nos ha permitido elaborar el Gráfico 1 en el que se muestran los porcentajes que los usuarios dan a cada aplicación teniendo en cuenta el sistema al que ya hemos aludido de cinco estrellas como puntuación máxima y una como la mínima. A través de él podemos observar que la aplicación que recibe más valoraciones positivas es Mondly con un $81 \%$ seguida de Memrise y Duolingo con un 80 y $78 \%$ respectivamente. A tenor de estos datos, podemos colegir que el número de descargas no se relaciona con la satisfacción que genera en los usuarios, pues la aplicación menos descargada es la que, en proporción, más satisface a los usuarios.

Gráfico 1- Porcentajes de las valoraciones que dan los usuarios por aplicación.

\begin{tabular}{|c|c|c|c|c|c|c|}
\hline \multirow{10}{*}{$\begin{array}{l}80 \\
70 \\
60 \\
50 \\
40 \\
30 \\
20 \\
10\end{array}$} & & & & & & \\
\hline & & & & & & \\
\hline & & & & & & \\
\hline & & & & & & \\
\hline & & & & & & \\
\hline & & & & & & \\
\hline & & & & & & \\
\hline & & & & & & \\
\hline & & & & & & \\
\hline & Duolingo & Babbel & Memrise & $\begin{array}{c}50 \text { Langua- } \\
\text { ges }\end{array}$ & Busuu & Mondly \\
\hline 5 estrellas & 78 & 60 & 80 & 68 & 62 & 81 \\
\hline 4 estrellas & 17 & 18 & 16 & 14 & 20 & 13 \\
\hline - 3 estrellas & 3 & 7 & 2 & 7 & 8 & 3 \\
\hline - 2 estrellas & 1 & 4 & 1 & 4 & 3 & 1 \\
\hline 1 estrella & 1 & 11 & 1 & 7 & 7 & 2 \\
\hline
\end{tabular}


Fuente: Elaboración propia.

Tras instalar las seis aplicaciones en un dispositivo móvil, hemos podido comprobar que todas consideran la posibilidad de implicar a otras personas en el proceso de aprendizaje pero en diferente grado. Esta implicación puede ir desde una notificación, esto es, no se espera una interacción, es simplemente una expresión de un hecho, a una auténtica interacción en la que los participantes pueden comunicarse intercambiando los roles de emisor y receptor.

En el caso de las notificaciones, expresión, hemos de destacar que en su mayoría se hacen en la lengua en la que es competente el aprendiz aunque, en algunos casos existe la posibilidad de expresarse también en otras. Las notificaciones pueden darse bien con los propios gestores de la aplicación, bien con otros usuarios y/o contactos que no están en la aplicación. Respecto a las primeras, las que se dan con los gestores, hay notificaciones que son desde la aplicación al usuario y otras desde el usuario a la aplicación. Se emplean para tratar diferentes asuntos, algunos relacionados con el propio proceso de aprendizaje y manejo de la aplicación, como mandar recordatorios de aprendizaje, explicar el uso de la aplicación o informar de que tenemos nuevos seguidores (que serían ejemplos de notificaciones que van desde la aplicación al usuario), y en otras ocasiones para abordar cuestiones relacionadas con los contenidos de la lengua meta como la opción que da Duolingo de transmitir que hemos encontrado un problema o error al hilo de alguno de los ejemplos que la aplicación incluye en sus ejercicios (que sería un ejemplo de notificación del usuario a los gestores de la aplicación). En este último caso, vemos que el usuario contribuye a la mejora de la aplicación y de ello se benefician todos los usuarios, esta idea puede relacionarse con las características del aprendizaje cooperativo de la interdependencia positiva y la responsabilidad del usuario con su papel dentro del grupo. No es difícil pensar que en la medida que el usuario puede acceder gratuitamente a unos contenidos seleccionados, graduados y estructurados, éste quiera contribuir de manera más o menos altruista a la mejora de la aplicación y que además el usuario pueda considerar que otros han hecho lo mismo o pueden hacerlo por él con otros ejemplos. Desde este punto de vista, la mejora de la aplicación es deber de todos, pues todos se nutren de ella.

También podemos observar que hay diferentes finalidades en los comentarios o notificaciones que se dan con otros usuarios. Entre esas finalidades encontramos, por ejemplo, la posibilidad de que el usuario agregue a amigos a la aplicación mediante el envío de enlaces o, la opción que más hemos encontrado en las seis aplicaciones analizadas, que el usuario 
comparta, normalmente en la lengua en la que se declara competente, la puntuación que ha alcanzado con personas desconocidas y/o conocidas, que pueden estar en la aplicación (usuarios) y/o no. En estos casos el contenido de estas notificaciones no se relaciona tanto con el aprendizaje de contenidos de la lengua, como sí con otras cuestiones que pueden afectar al proceso de aprendizaje. Pero no siempre es así, hay otras notificaciones, en este caso nos referimos a los wikis, que sí tienen que ver con los contenidos de la lengua meta, en concreto hablamos de los "mem" de Memrise, que son ideas sobre el objeto de estudio, la lengua meta, que los aprendices cuelgan. Es cierto que no existe en estos wikis una idea de interdependencia positiva, ya que aunque algunos usuarios no creen ninguno, pueden acceder a los que otros han aportado y beneficiarse de ellos, por otro lado, tampoco hay interacción, pues quienes deciden colgarlos asumen que no habrá respuesta alguna por parte de otros miembros, entonces ¿dónde puede estar la recompensa a este esfuerzo?, muy probablemente en ayudarse a uno mismo a memorizar y en pensar que nuestro "mem" también puede ayudar a otros a mejorar en el conocimiento de la lengua meta y que nosotros también podemos beneficiarnos de los "mem" de otros usuarios.

Además de las notificaciones, las aplicaciones permiten la interacción con los gestores de la aplicación y entre los usuarios. Dentro del primer caso, nos encontramos una interacción que parte del usuario hacia los gestores de la aplicación, suele emplearse este procedimiento para solicitar un tipo de ayuda o para hacer consultas. En todos los casos encontrados, se trata de una comunicación asincrónica o no simultánea, en la que el usuario explica algo y espera una respuesta por parte de los gestores de la aplicación, aunque ésta no siempre se da. Es muy probable que esta interacción no tenga que ver con los contenidos de la lengua meta, como sucede con las consultas de Babbel, por ejemplo, aunque tampoco se podrían descartar.

La interacción con otros usuarios está presente en varias aplicaciones, uno de los ejemplos más claros es la manera en la que se gestionan los ejercicios de escritura en Busuu. Estos ejercicios de escritura permiten al usuario actuar como "experto" y sentir que contribuye a la mejora de la competencia comunicativa de otros para que los demás puedan hacer lo mismo con la suya. Al igual que sucedía con los “mem”, este ayudar a aprender nos parece que se relaciona con el aprendizaje cooperativo pero también con la telecolaboración7.

\footnotetext{
${ }^{7}$ En palabras de Jauregi (2015, p. 79) la telecolaboración consiste: “en ofrecer la posibilidad a nuestros alumnos de que colaboren en proyectos con alumnos de otras instituciones en el extranjero a través de aplicaciones digitales para así desarrollar no sólo su habilidad comunicativa e intercultural sino también las destrezas de alto rango cognitivo".
} 
Es cierto que en general no son muchas las posibilidades de interacción que hay en los ejercicios de las aplicaciones, algo fundamental para el aprendizaje de lenguas, y que los ejercicios de escritura de Busuu pueden además cosechar correcciones imprecisas, incorrectas, no siempre comprensibles o ajustadas al nivel de comprensión del alumno que es corregido ni ajustadas a las normas lingüísticas, ya que el hecho de ser competente en una lengua, especialmente si se es nativo, no garantiza que se sepa enseñar esa lengua ni que se sea consciente de las normas de uso que la sustentan, pero su valor es, a nuestro modo de ver, incuestionable, por cuanto estas explicaciones que pueden ofrecer los hablantes más competentes (incluidos nativos) al aprendiz, si están hechas desde la lengua meta, son muestras de lengua (inputo aducto) reales, que le obligan a tratar de comprender y le ofrecen la posibilidad de entrar en contacto con nativos de la lengua meta, con quienes poder practicar la lengua en contextos sociales auténticos y variados y que a su vez pueden estar interesados en aprender la lengua del primer usuario, para seguir desarrollando nuevas actividades dentro y fuera de la aplicación, como puede ser un trabajo de tándem.

Existen algunos "espacios" que pueden ser utilizados por los usuarios de ambas maneras, bien para interactuar, bien para colgar comentarios, en Duolingo encontramos varios ejemplos: el foro, los comentarios que aparecen mediante el símbolo de un bocadillo de cómic surgidos al hilo de cualquier ejemplo de cada ejercicio que la aplicación incluya o los clubes. En ellos mayoritariamente los usuarios reflexionan sobre la lengua, quienes más saben, ofrecen a otros sus conocimientos, algo que encaja con el propósito perseguido por el aprendizaje cooperativo, según Cassany (2009), de fomentar la cooperación y una enseñanza reflexiva que se base en el desarrollo de habilidades y no tanto en la memorización.

\section{CONCLUSIONES}

Podemos concluir este trabajo afirmando que trabajar un idioma con aplicaciones no es sinónimo de trabajarlo en soledad, ya que en las aplicaciones de idiomas se puede incluir a otros sujetos, conocidos previamente o no, la comunicación con ellos así como la cooperación entre ellos, pero la presencia de todos estos factores es desigual en las distintas aplicaciones. Pese a todo, hay una tendencia a considerarlos, de hecho algunas aplicaciones incluyen publicidad en esta línea para conseguir más usuarios, aunque no siempre después se cumpla.

Por otro lado, la cooperación puede no coincidir exactamente con todas las características que hemos mencionado en este trabajo del aprendizaje cooperativo, pero sí 
pueden apreciarse algunas de ellas, como cierta forma de interdependencia positiva, la interacción o la responsabilidad de cada miembro. También es posible que ninguna de las aplicaciones encaje de manera obvia y completa en los métodos del aprendizaje cooperativo, aunque pueden aparecer técnicas relacionadas y pueden surgir otras formas de colaboración como la telecolaboración.

De todos modos, el análisis que hemos presentado ha de ser tomado con cierta cautela, pues nosotros hemos considerado estas aplicaciones en abstracto, es decir, sin enmarcarlas dentro del contexto formal de enseñanza de idiomas, donde contamos con el grupo clase y donde también se podrían incorporar como material. En este sentido nos hacemos eco de las palabras de Jordano de la Torre, Castrillo de Larreta Azelaín y Pareja Lora (2016, p. 31) quienes reconocen que: "el verdadero valor pedagógico y educativo de estos materiales para el aprendizaje de lenguas a través de dispositivos móviles viene determinado en muchas ocasiones por el uso que el docente haga de los mismos en el aula, y por la metodología que se siga".

Por otro lado, tal y como ya reconocíamos en la introducción de este trabajo, este es un campo en constante reestructuración y crecimiento que aún puede variar mucho, en palabras de Jordano de la Torre et al. (ibídem) "este tipo de aplicaciones se encuentra en una fase muy inicial de desarrollo y no han alcanzado aún ni la calidad ni la madurez pedagógica o metodológica de las que gozan otros materiales asociados a prácticas docentes más tradicionales". De manera que habrá que darle tiempo al tiempo, para extraer conclusiones definitivas.

\section{REFERENCIAS}

CASSANY, Daniel. La cooperación en ELE: de la teoría a la práctica. TINKUY 11, pp. 7-29, 2009. Disponible en: https://dialnet.unirioja.es/servlet/articulo?codigo=3303819 Acceso en marzo de 2017.

JAUREGI, Kristi. La telecolaboración como estrategia de innovación didáctica de ELE en el siglo XXI. Mosaico 33, pp. 74-91, 2015. Disponible en: https://sede.educacion.gob.es/publiventa/mosaicon-33-revista-para-la-promocion-y-apoyo-a-la-ensenanza-del-espanol/ensenanza-lenguaespanola/20629 Acceso en marzo de 2017.

JOHNSON, David W., JOHNSON Roger T. y HOLUBEC, Edythe J. El aprendizaje cooperativo en el aula. Buenos Aires: Paidós, 1999.

JOHNSON, David W., JOHNSON, Roger T. y STANNE, Mary Beth. Cooperative Learning methods: A Meta-Analysis, 2000. Disponible en: https://www.researchgate.net/profile/David_Johnson50/publication/220040324_Cooperative_learning _methods_A_meta-analysis/links/00b4952b39d258145c000000.pdf Acceso en marzo de 2017. 
JORDANO DE LA TORRE, María, CASTRILLO DE LARRETA AZELAÍN, María Dolores y PAREJA LORA, Antonio. El aprendizaje de lenguas extranjeras mediante la tecnología móvil en el contexto de la educación a distancia y combinada. RIED 19 (1), pp. 25-40, 2016. Disponible en: http://e-spacio.uned.es/fez/eserv/bibliuned:revistaRied-2016-19-1-

7010/Aprendizaje_de_lenguas_extranjeras.pdf Acceso en marzo de 2017.

OLSEN, R.E.W.B. y KAGAN, Spencer. About cooperative learning, en Kessler, Carolyn. (Ed.)

Cooperative language learning: A teacher's resource book. Englewood Cliffs, NJ: Prentice Hall, pp. 1-30, 1992.

OVEJERO BERNAL, Anastasio. El aprendizaje cooperativo. Una alternativa eficaz a la enseñanza tradicional. Barcelona: PPU, 1990.

SLAVIN, Robert E. Cooperative learning. Theory, research, and practice. Needham Heights, MA: Allyn and Bacon, 1990.

SMITH, Karl. Cooperative learning: Making "groupwork" work. New directions for teaching and learning, vol. 67, pp. 71-82, 1996.

Webs

https://www.duolingo.com/help Acceso en marzo de 2017.

https://home.babbel.com/dashboard Acceso en marzo de 2017.

https://play.google.com/store/apps?hl=es Acceso en marzo de 2017.

Aplicaciones

Babbel

Busuu

Duolingo

Memrise

Mondly

50 Languages 
Ana María Aguilar López

Miembro del grupo de investigación Desarrollo personal, liderado por la Dra. Beatriz Peña Acuña. Profesora del Área de Didáctica de la Lengua y la Literatura de la Universidad de Burgos (España). Doctora en 2012 con la tesis Influencia de la dramatización en la expresión oral de discentes universitarios extranjeros y nativos. Profesora de Español Lengua

Extranjera en la Universidade Estadual do Rio Grande do Norte (Brasil), lectora oficial del

Ministerio de Asuntos Exteriores de España en la Jihočeská Univerzita (República Checa).

Profesora de Español Segunda Lengua en la Universidad de Burgos y el Instituto de la lengua castellano y leonés. Coautora del Método de español para extranjeros Hablamos nivel C.

Formadora de profesorado en la Ruhr Universität Bochum (Alemania) y en cursos organizados por el Instituto de la lengua castellano y leonés y el Instituto Cervantes.

Esta obra está licenciada sob uma Licença Creative Commons. 